\title{
Expression of gallus epidermal growth factor (gEGF) with food-grade Lactococcus lactis expression system and its biological effects on broiler chickens
}

\section{Yu Zhou}

Huazhong Agriculture University

\section{Pinpin Chen}

Huazhong Agriculture University

\section{Shuai Shi}

Huazhong Agriculture University

\section{Xiaowen Li}

Huazhong Agriculture University

\section{Deshi Shi}

Huazhong Agriculture University

\section{Zutao Zhou}

Huazhong Agriculture University

Zili Li

Huazhong Agriculture University

Yuncai Xiao ( $\nabla$ xyc88@mail.hzau.edu.cn )

HUAZHONG AGRICULTURAL UNIVERSITY https://orcid.org/0000-0002-6945-1497

\section{Research article}

Keywords: gallus epidermal growth factor, Lactococcus lactis, broiler chickens, growth performance, immune function, intestinal development

Posted Date: June 3rd, 2020

DOI: https://doi.org/10.21203/rs.2.18682/v2

License: (c) (1) This work is licensed under a Creative Commons Attribution 4.0 International License. Read Full License 
Version of Record: A version of this preprint was published at Biomolecules on January 14th, 2021. See the published version at https://doi.org/10.3390/biom11010103. 


\section{Abstract}

Background Epidermal growth factor (EGF) is a multifunctional polypeptide, which could be utilized to solve problems in the industry such as increasing growth performance of commercial broilers or enhancing resistance to diseases under adverse conditions. Thus far, very few studies have focused on the gallus epidermal growth factor (gEGF) despite the availability of a plethora of studies on mammalian EGF. The aim of this study was to express gallus epidermal growth factor (gEGF) using a food-grade Lactococcus lactis expression system and to investigate its biological effects on broiler chickens.

Results A recombinant Lactococcus lactis, which produced the secretary form of bioactive gEGF at 2.67 $\mu \mathrm{g} / \mathrm{mL}$ in culture supernatant, was generated. In vitro testing denoted that gEGF promoted the proliferation of UMNSAH/DF-1 cells. Sixty-three 5-day-old broiler chickens were divided into three groups and treated with either M17 medium (C, as control), supernatant of LL-pNZ8149 fermentation product (PLL), or supernatant of LL-pNZ8149-gEGF fermentation product (gEGF-P-LL). Body weight (BW), average daily gain (ADG), and the gain:feed ratio of the gEGF-P-LL group were significantly higher than those of the other groups during the two-week study. In addition, two weeks after therapy, the indices of spleen and thymus gland, levels of serum IgA, IgG and duodenum mucosal slgA were all significantly higher in the gEGF-P-LL group than in the other groups. Moreover, the villus height of the duodenum, jejunum and ileum and crypt depth of the jejunum of the gEGF-P-LL group were significantly higher than those of the other groups, while the crypt depth of the duodenum showed the opposite pattern during the two-week study.

Conclusions The bioactive gEGF could be expressed with Lactococcus lactis expression system. Furthermore, gEGF has the potential to enhance growth performance, immune function, and intestinal development in broiler chickens.

\section{Background}

Epidermal growth factor (EGF) is a small polypeptide that can promote cell proliferation and differentiation [1]. EGF has been shown to play an important role in gastrointestinal repair [2], gastric acid secretion [3], the regulation of embryo development [4,5], and the regulation of ion transport [6,7]. Recent studies have shown that EGF and EGFR may also be involved in tumorigenesis [8,9], and EGFR has been utilized as a target for cancer therapy. Thus, EGF is an influential molecule involved in multiple biological processes. Epidermal growth factor is produced using genetic techniques, such as expression in E. coli [10,11], Bacillus brevis [12,13], or Pichia pastoris [14,15].

The biological effects of EGF on animals have also been investigated. The growth performance of earlyweaned pigs was enhanced by feeding them with a fermentation product of EGF-expressing Lactococcus lactis [16]. Dietary supplementation with pEGF enhanced daily weight gain for $0-7$ days postweaning, significantly increased IgA serum levels at day 18 postweaning, and significantly increased both mucosal IgA levels and crypt depth in the jejunum at day 28 postweaning, indicating that EGF can promote growth 
performance and immune function [17]. Further, piglet diets supplemented with EGF can enhance protection against intestinal pathogens $[18,19]$ and promote intestinal repair after rotavirus infection [20]. EGF can also improve growth prior to challenge with Eimeria and improve gut function indices upon Eimeria challenge [21].

However, very few studies have focused on gallus epidermal growth factor (gEGF) and its biological activity. In the current market, it is imperative to improve the growth performance of young commercial broilers in the absence of antibiotics, as these drugs have recently been banned from production. Further, it is important to improve the resilience of chickens to adverse conditions, such as temperature or stress. Moreover, based on the positive effects of EGF in early-weaned piglets [16] and rats [2], it is important to investigate the biological effects of gEGF on chickens. In this study, a recombinant $L$. lactis strain that secretes gEGF (LL-pNZ8149-gEGF) was constructed. Due to concerns regarding the risk associated with the use of genetically modified organisms, the supernatant of the LL-pNZ8149-gEGF fermentation product was used to treat broilers instead of the direct use of EGF (without LL-pNZ8149-gEGF), and the growth performance, immune function, and intestinal development of these broilers were assessed. This study serves as a reference for the application of gEGF in broiler breeding.

\section{Results}

\section{Expression of gEGF protein in a bacterial culture supernatant}

Both LL-pNZ8149 and LL-pNZ8149-gEGF transformants were induced with $10 \mathrm{ng} / \mathrm{mL}$ nisin for $6 \mathrm{~h}$, and the supernatants were collected for western blot analysis. Figure 1 depicts the protein from the LLpNZ8149-gEGF fermentation product hybridized with the His-tag monoclonal antibody (Abbkine Scientific Co., Ltd.), indicating that it contained the target protein.

The expression of gEGF in the supernatant of the LL-pNZ8149-gEGF fermentation product induced with different concentrations of nisin and different induction times is shown in Figure 2 and Figure 3 , respectively. As nisin concentration increased from $0-40 \mathrm{ng} / \mathrm{mL}$, the expression of gEGF initially increased but then decreased at $10 \mathrm{ng} / \mathrm{mL}$. A similar pattern was observed as induction time increased from $0-24 \mathrm{~h}$, with the expression of gEGF initially increasing and then decreasing at $12 \mathrm{~h}$.

Figure 4 depicts the results of an SDS-PAGE analysis of the purified protein from the supernatant of the LL-pNZ8149-gEGF fermentation product. The band near $7 \mathrm{kDa}$ corresponded to the computed MW of 
gEGF. The protein concentration of the $5 \mathrm{~mL}$ purified protein was $0.16 \mathrm{mg} / \mathrm{mL}$, as determined by the BCA protein assay kit; in other words, the concentration of gEGF in the supernatant of LL-pNZ8149-gEGF fermentation product was $2.67 \mu \mathrm{g} / \mathrm{mL}$, and Figure 5 depicts the standard curve for bovine serum albumin.

\section{gEGF promotes UMNSAH/DF-1 cell proliferation}

Figure 6 shows the effect of $1-20 \mu \mathrm{g} / \mathrm{mL}$ of gEGF on UMNSAH/DF-1 cell proliferation. UMNSAH/DF-1 cell proliferation was significantly enhanced after treatment with 1,5 , and $10 \mu \mathrm{g} / \mathrm{mL}$ of gEGF. The strongest effect was observed for $5 \mu \mathrm{g} / \mathrm{mL}$ of gEGF.

\section{gEGF promotes the growth performance of broilers}

In contrast to the Control group and the P-LL group, the body weight (BW) in the gEGF-P-LL group significantly increased after 1 week (gEGF-P-LL v.s. C,gEGF-P-LL v.s. P-LL,) and 2 weeks (gEGF-P-LL v.s. C,gEGF-P-LL v.s. P-LL,), as did the average daily feed intake (ADFl; gEGF-P-LL v.s. C,gEGF-P-LL v.s. PLL,gEGF-P-LL v.s. C,gEGF-P-LL v.s. P-LL,) and the average daily gain (ADG; gEGF-P-LL v.s. C, $P<0.01$; gEGF-P-LL v.s. P-LL,gEGF-P-LL v.s. C,gEGF-P-LL v.s. P-LL,. Further, in contrast to the Control group, the gain:feed ratio in the P-LL group and the gEGF-P-LL group increased significantly in the first week gEGF-P-

LL v.s. C,P-LL v.s. $C_{\text {n }}$ although there were no significant differences between these two groups . At the conclusion of the two-week study, the gain:feed ratio of the gEGF-P-LL group significantly increased compared with that of the other two groups gEGF-P-LL v.s. C,gEGF-P-LL v.s. P-LL,.

Table 1. Effects of gEGF on the growth performance and feed efficiency of broilers. 


\begin{tabular}{|c|c|c|c|c|c|c|}
\hline \multirow[t]{2}{*}{ Item } & & \multicolumn{3}{|c|}{ Treatment } & \multirow[t]{2}{*}{ SEM } & \multirow[t]{2}{*}{ P-value } \\
\hline & & C & P-LL & gEGF-P-LL & & \\
\hline IBW (g) & & 91.43 & 92.00 & 91.42 & 1.79 & 0.930 \\
\hline \multirow[t]{2}{*}{$\mathrm{BW}(\mathrm{g})$} & Week 1 & $210.47^{a}$ & $225.52^{\mathrm{a}}$ & $238.62^{b}$ & 5.62 & 0.018 \\
\hline & Week 2 & $395.14^{\mathrm{a}}$ & $390.19^{a}$ & $442.10^{b}$ & 12.46 & 0.006 \\
\hline \multirow[t]{2}{*}{ ADFI (g) } & Week 1 & $30.43^{a}$ & $31.12^{\mathrm{a}}$ & $33.57^{b}$ & 0.71 & 0.011 \\
\hline & Week 2 & $33.12^{\mathrm{a}}$ & $32.65^{\mathrm{a}}$ & $36.40^{\mathrm{b}}$ & 1.02 & 0.020 \\
\hline \multirow[t]{2}{*}{$\operatorname{ADG}(g / d)$} & Week 1 & $17.01^{a}$ & $19.10^{\mathrm{a}}$ & $21.03^{b}$ & 0.67 & 0.007 \\
\hline & Week 2 & $26.59^{a}$ & $26.08^{a}$ & $29.87^{b}$ & 1.32 & 0.018 \\
\hline \multirow[t]{2}{*}{ Gain:feed $(\mathrm{g} / \mathrm{g})$} & Week 1 & $0.56^{\mathrm{a}}$ & $0.61^{b}$ & $0.63^{b}$ & 0.17 & 0.016 \\
\hline & Week 2 & $0.80^{\mathrm{a}}$ & $0.80^{\mathrm{a}}$ & $0.82^{b}$ & 0.06 & 0.027 \\
\hline
\end{tabular}

SEM: Standard Error of Mean;

Data represented mean of 3 replications per treatment with 7 broilers per replication $(n=3)$.

Means within a row without superscripts are of no significant difference $(p>0.05)$.

a,b Means within a row without common superscripts differ significantly $(p<0.05)$.

IBW: initial body weight; BW: body weight; ADFI: average daily feed intake; ADG: average daily gain.

\section{gEGF improves the immune function of broilers}

Table 2 shows the effect of gEGF on the index of different immune organs from the treated broilers. Compared with those of the Control group and the P-LL group, the indices of spleen gEGF-P-LL v.s. C,gEGF-P-LL v.s. P-LL,Table 2 and thymus gland gEGF-P-LL v.s. C,gEGF-P-LL v.s. P-LL,Table 2 in the gEGFP-LL group increased significantly after two weeks Further, the BF Index of the gEGF-P-LL group was significantly higher than that of the P-LL group Table 2, and the BF Index of the gEGF-P-LL group was 
higher than that of the Control group, although this difference was not statistically significant for the twoweek study Table 2 .

Table 2. Effect of gEGF on the index of immune organs of treated broilers following two weeks of treatment.

\begin{tabular}{ccccccc}
\hline \multirow{2}{*}{ Item } & \multicolumn{3}{c}{ Treatment } & \multirow{2}{*}{ SEM } & P-value \\
\cline { 2 - 4 } & C & P-LL & gEGF-P-LL & & \\
\hline Spleen weight (g) & $0.38^{\mathrm{a}}$ & $30.38^{\mathrm{a}}$ & $0.57^{\mathrm{b}}$ & 0.28 & 0.001 \\
BW (g) & $397.11^{\mathrm{a}}$ & $395.34^{\mathrm{a}}$ & $446.67^{\mathrm{b}}$ & 4.29 & $<0.001$ \\
Index of spleen & $0.95^{\mathrm{a}}$ & $0.97^{\mathrm{a}}$ & $1.28^{\mathrm{b}}$ & 0.06 & 0.002
\end{tabular}

pleen weight $(\mathrm{mg}) / \mathrm{BW}(\mathrm{g}))$

BF weight (g)

BW $(g)$

Index of BF

$1.43^{\mathrm{a}} \quad 1.31^{\mathrm{a}}$

$397.11^{\mathrm{a}} \quad 395.34^{\mathrm{a}}$

$3.59^{\mathrm{ab}}$

$3.30^{\mathrm{a}}$ $1.69^{b}$

$0.62 \quad 0.002$

$446.67^{b}$

$4.29<0.001$

$3.78^{b}$

$0.14 \quad 0.040$

(BF weight (mg)/BW (g))

$\begin{array}{cccccc}\text { TG weight (g) } & 2.18^{\mathrm{a}} & 2.24^{\mathrm{a}} & 2.86^{\mathrm{b}} & 0.77 & <0.001 \\ \text { BW (g) } & 397.11^{\mathrm{a}} & 395.34^{\mathrm{a}} & 446.67^{\mathrm{b}} & 4.29 & <0.001 \\ \text { Index of TG } & 5.50^{\mathrm{a}} & 5.66^{\mathrm{a}} & 6.40^{\mathrm{b}} & 0.20 & 0.008\end{array}$

(TG weight (mg)/BW (g))

SEM: Standard Error of Mean; BF: bursa of Fabricius; TG: Thymus gland.

Data represented mean of 3 replications per treatment with 3 broilers per replication $(n=3)$.

Means within a row without superscripts are of no significant difference $(p>0.05)$.

a,b Means within a row without common superscripts differ significantly $(p<0.05)$. 
Table 3 shows the effect of gEGF on serum and mucosa immunoglobulins of the treated broilers after two weeks. The concentration levels of serum IgA gEGF-P-LL v.s. C,gEGF-P-LL v.s. P-LL,Table 3 and IgG gEGF-P-LL v.s. C,gEGF-P-LL v.s. P-LL,Table 3 in the gEGF-P-LL group were significantly higher than those in the Control and P-LL groups. Further, duodenum mucosa slgA increased significantly in the gEGF-P-LL groupgEGF-P-LL v.s. C,gEGF-P-LL v.s. P-LL,Table 3.

Table 3. Effect of gEGF on serum and mucosal immunoglobulins of treated broilers after two weeks of treatment.

\begin{tabular}{ccccccc}
\hline \multirow{2}{*}{ Item } & & \multicolumn{3}{c}{ Treatment } & \multirow{2}{*}{ SEM } & P-value \\
\cline { 3 - 5 } & & $\mathrm{C}$ & P-LL & gEGF-P-LL & \\
\hline \multirow{2}{*}{ Serum } & $\operatorname{lgA}(\mathrm{mg} / \mathrm{mL})$ & $0.27^{\mathrm{a}}$ & $0.23^{\mathrm{a}}$ & $0.30^{\mathrm{b}}$ & 0.01 & 0.004 \\
& $\operatorname{lgG}(\mathrm{mg} / \mathrm{mL})$ & $4.07^{\mathrm{a}}$ & $3.96^{\mathrm{a}}$ & $5.09^{\mathrm{b}}$ & 0.34 & 0.010 \\
& & & & & & \\
Mucosa slgA (ng/mg) & Duodenum & $197.20^{\mathrm{a}}$ & $193.80^{\mathrm{a}}$ & $371.34^{\mathrm{b}}$ & 8.13 & $<0.001$ \\
& Jejunum & 226.65 & 247.90 & 219.22 & 26.27 & 0.559 \\
& Ileum & 619.85 & 603.26 & 640.77 & 26.82 & 0.428 \\
\hline
\end{tabular}

SEM: Standard Error of Mean;

Data represented mean of 3 replications per treatment with 3 broilers per replication $(n=3)$.

Means within a row without superscripts are of no significant difference $(p>0.05)$.

$a, b$ Means within a row without common superscripts differ significantly $(p<0.05)$.

gEGF promotes the intestinal development of broilers 
Table 4 illustrates the effect of gEGF on the mucosal morphological traits of the small intestine of broilers after two weeks of treatment. The villus heights of the duodenum gEGF-P-LL v.s. C,gEGF-P-LL v.s. P-LL,Table 4, jejunum gEGF-P-LL v.s. C,gEGF-P-LL v.s. P-LL,Table 4 and ileum gEGF-P-LL v.s. C,gEGF-P-LL v.s. P-LL,Table 4 in the gEGF-P-LL group were significantly higher than those in the Control and P-LL groups. The crypt depth of the jejunum gEGF-P-LL v.s. C,gEGF-P-LL v.s. P-LL,Table 4 in the gEGF-P-LL group was significantly higher than that of the other groups, while the crypt depth of the duodenum in the gEGF-P-LL group were lower than those in the P-LL group Table 4Table 4. Further, the gEGF treatment significantly increased the VH/CD of the duodenum gEGF-P-LL v.s. C,gEGF-P-LL v.s. P-LL,Table 4, but not that of the jejunum gEGF-P-LL v.s. C,gEGF-P-LL v.s. P-LL,Table 4 and ileum gEGF-P-LL v.s. C,gEGF-P-LL v.s. P-LL,Table 4.

Table 4. Effect of gEGF on the mucosal morphological traits of the small intestine of treated broilers after two weeks of treatment. 


\begin{tabular}{|c|c|c|c|c|}
\hline \multirow[t]{2}{*}{ Item } & \multicolumn{3}{|c|}{ Treatment } & \multirow[t]{2}{*}{ SEM P-value } \\
\hline & C & P-LL & gEGF-P-LL & \\
\hline \multicolumn{5}{|l|}{ Duodenum } \\
\hline Villus height & $1116.11^{\mathrm{a}}$ & $1210.01^{a}$ & $1333.72^{b}$ & 41.850 .006 \\
\hline Crypt depth & $97.17^{a b}$ & $97.08^{a}$ & $84.79^{b}$ & $6.32 \quad 0.016$ \\
\hline $\mathrm{VH} / \mathrm{CD}$ & $11.59^{a}$ & $12.47^{\mathrm{a}}$ & $15.73^{b}$ & $0.64 \quad 0.001$ \\
\hline \multicolumn{5}{|l|}{ Jejunum } \\
\hline Villus height & $763.91^{a}$ & $757.41^{a}$ & $951.30^{\mathrm{b}}$ & $16.90<0.001$ \\
\hline Crypt depth & $97.63^{a}$ & $100.03^{a}$ & $130.51^{b}$ & $\begin{array}{ll}7.31 & 0.007\end{array}$ \\
\hline $\mathrm{VH} / \mathrm{CD}$ & 7.87 & 7.62 & 7.29 & $0.47 \quad 0.504$ \\
\hline \multicolumn{5}{|l|}{ Ileum } \\
\hline Villus height & $652.11^{a}$ & $648.67^{a}$ & $695.15^{b}$ & 13.720 .026 \\
\hline Crypt depth & 82.94 & 85.01 & 88.47 & $6.07 \quad 0.673$ \\
\hline $\mathrm{VH} / \mathrm{CD}$ & 7.86 & 7.72 & 7.87 & $0.50 \quad 0.949$ \\
\hline
\end{tabular}

SEM: Standard Error of Mean;

Data represented mean of 3 replications per treatment with 3 broilers per replication $(n=3)$.

Means within a row without superscripts are of no significant difference $(p>0.05)$.

a,b Means within a row without common superscripts differ significantly $(p<0.5)$.

\section{Discussion}

EGF has been considered a powerful bioactive molecule since it was first discovered in 1962; however, initial extraction methods resulted in low yields. Today, this is no longer the case as EGF is mass produced by utilizing a variety of expression systems. However, results from other studies [10, 12-16, 22] have revealed that different types of systems yield remarkably different expression levels of EGF, and even when utilizing the same system, levels of EGF can vary depending on the conditions. In addition, protein production in nisin-induced $L$. lactis expression systems could be increased by optimizing various factors [23]. In the present study, gEGF was expressed with a nisin-induced L. lactis expression system, 
and nisin concentration and induction time were optimized for yield. Our results showed that both the concentration of nisin and induction time could affect the expression level of gEGF. As the concentration of nisin increased, the gEGF yield increased initially, followed by a decrease at $10 \mathrm{ng} / \mathrm{mL}$. While a threshold concentration of nisin is required for complete induction, excess concentrations could inhibit the growth of the host strain due to innate antibacterial properties. Further, as the induction time increased, the expression level of EGF increased gradually before decreasing at $12 \mathrm{~h}$, perhaps due to excess lactate accumulation, which disrupts cell metabolism, during cell growth $[24,25]$. The concentration of gEGF in the supernatant was $2.67 \mu \mathrm{g} / \mathrm{mL}$, which was similar to or higher than the results of other studies $[12,14,16,22]$.

EGF promotes cell proliferation and differentiation. Despite minor differences in the amino acid sequences of EGF from different species, similarity of the EGF receptor (EGFR) structure ensures that EGF is not highly species-specific. For example, hEGF has been shown to function in rats [26], mEGF in pigs [27], and pEGF in chickens [21], although some anomalies have been reported. Lax et al. showed that the binding ability of mEGF to chicken EGFR was 100 times lower than that to human EGFR; further, the binding ability of TGF-alpha to chicken EGFR was equal to or higher than that to human EGFR. These results highlight the differences in the binding ability of EGF compared to that of EGFR in different species [28], indicating that EGF of a given species may have different effects for another species. The proliferation of human dermal fibroblasts has been shown to be promoted by hEGF [29]. Therefore, in the current study, a chicken embryo fibroblast line, UMNSAH/DF-1, was used to detect the biological activity of gEGF in vitro. Our results showed that gEGF could promote the proliferation of DF1 cells. Moreover, the ability to promote proliferation increased initially with gEGF concentration before decreasing at $5 \mu \mathrm{g} / \mathrm{mL}$, indicating that the proliferation-promoting effect of gEGF was concentration-dependent.

EGF has been reported to promote growth performance, improve immune function, and stimulate intestinal development or repair in animals $[16,17,30,31]$. Wang et al. showed that 14 days of rearing early-weaned piglets with LL-pEGF significantly increased the final body weight and average daily gain of these piglets, compared to the control group [31]. Bedford et al. showed that the supernatant of recombinant Lactococcus lactis could promote growth performance in early-weaned piglets, while the fermentation product, which expressed EGF, could not, possibly due to bacterial overload [16]. However, since previous researches have focused on EGF in mammals [17,32,33], there are no studies concerning gEGF or its biological activity. Therefore, it was necessary and meaningful to investigate these activities in relation to growth performance, immune function, and the intestinal development of broilers.

The effect of gEGF on the immune function of broilers was evaluated by measuring the immune organ index, serum IgA and IgG, and intestinal mucosal slgA. Very few studies have investigated the effects of 
EGF on immune function. Dietary supplementation of recombinant pEGF was shown to effectively increase the level of IgA in the jejunum mucosa of weaned piglets [34-36]. Further, the level of serum IgA in weaned piglets increased significantly after 18 days of treatment with pEGF, while the same treatment significantly increased levels of IgA in the jejunum mucosa after 28 days [17]. In our study, serum IgA increased significantly during 2 weeks of gEGF treatment, which was similar to the effect of pEGF in piglets. However, the level of slgA only increased in the mucosa of the duodenum, and not in the mucosa of the jejunum or ileum. This result may be due to degradation of gEGF along the gastrointestinal tract as degradation rates of EGF in the small intestinal lumen of weaned pigs has been shown to increase from the proximal to the mid and distal regions [37], which resulted in relatively high levels of gEGF in the proximal intestinal tract. In addition, we found that the thymus and spleen indices increased significantly in broilers treated with gEGF for 2 weeks, indicating that immune function improved compared to the phenotype. Overall, these results suggest that gEGF could improve immune function in broilers.

The effect of gEGF on the intestinal development of broilers was evaluated by measuring villus height, crypt depth, and VH/CD in the mucosa of the duodenum, jejunum, and ileum. Intestinal villus with greater height has a larger area for nutrient absorption, leading to enhanced absorption, while shallower crypts lead to higher maturation rates of intestinal epithelial cells and better absorption. The VH/CD value could thus reflect the function of the small intestine, with higher values implying better nutrient absorption in the intestine [38]. Our results clearly showed that gEGF promoted the development of the intestinal villus in the duodenum, jejunum, and ileum following 2 weeks of gEGF treatment; however, in the case of crypt depth, gEGF could only promote development in the duodenum. Thus, gEGF effectuated a pronounced effect on the villus, in contrast to the findings of Thompson et al. indicating that EGF had greater effects on the crypt [39]. Moreover, gEGF was found to be strongly associated with the development of the duodenum, which could also be due to varied degradation rates within different regions of the intestine [36].

This study evaluated growth performance by measuring the body weight, average daily gain, daily feed intake, and the gain:feed ratio. Most indices of growth performance were improved during the first week of gEGF treatment. The current study found that an increase in the gain:feed ratio occurred both in the PLL and gEGF-P-LL groups compared with that in the Control group within the first week, suggesting that there may be some components of the fermentation product of LL-pNZ8149 affecting growth performance. However, the gain:feed ratio of the gEGF-P-LL group was significantly higher than that of the other two groups following 2 weeks of gEGF treatment, indicating that gEGF was an important factor in the growth of broilers. In addition, these results indicated that gEGF significantly improved the growth performance of broilers via increasing the gain:feed ratio. Kim et al. showed that mEGF exerted beneficial effects on the growth performance of broiler chickens prior to challenge with Eimeria, while enhancing the expression of the nutrient transporter gene $x C T 1$ [21]. Bedford et al. found that the supernatant of the 
EGF-LL fermentation product increased levels of sucrase and alkaline phosphatase by day 8 of treatment [16]. Wang et al. reported that sucrase in 3 intestinal segments, aminopeptidase $A$ in the duodenum and jejunum, and aminopeptidase $\mathrm{N}$ and dipeptidase $\mathbb{\nabla}$ in the duodenum were significantly higher in the group treated with LL-pEGF than in the control group [30]. Thus, enhanced expression of nutrient transporters and increased levels of digestive enzymes caused by EGF may have contributed to the improved growth performance of broilers and early-weaned piglets. Bedford et al. showed that the gain:feed ratio of earlyweaned piglets did not increase following 2 weeks of treatment with the supernatant of recombinant bacteria [16]. Therefore, further study will be required to investigate if the growth- promoting effect of gEGF is transient. However, overall, our results suggested that gEGF may be utilized as a growth promotant in broiler chickens.

Antibiotics such as virginiamycin have been used as growth promotants in broiler chickens for decades [40] because initially, they inhibited pathogenic bacteria; reduced the use of nutrients by bacteria; increased the synthesis of vitamins and other growth factors; and promoted nutrient absorption by reducing the thickness of intestinal epithelial cells [41]. However, long-term use of antibiotics resulted in reduction of sensitivity to the drug, often leading to complete resistance to this therapy $[42,43]$. Moreover, continual use of antibiotics destroyed normal microbiota along with the targeted flora, causing serious damage to intestinal microecology $[44,45]$. In addition, abuse of antimicrobials in commercial animals could lead to finished livestock and poultry products containing antibiotic residue in the form of prototypes or metabolites, which might alter the intestinal flora of consumers or result in allergic reactions, threatening the health of consumers. At the same time, overuse of antibiotics increased the incidence of drug-resistant bacterial diseases in both humans and animals. Thus, the current study trialed gEGF as an alternative to antibiotics and found that this therapy significantly promoted the growth performance of broiler chickens, via increased food intake and weight gain, improved nutrient absorption, and enhanced intestinal and immune functioning. Therefore, gEGF should be trialed as an alternative growth promotant because it exhibited non-toxic side effects and did not elicit an antibacterial effect that could influence the natural intestinal flora of consumers, and thereby might avoid some of the problems associated with chicken and food safety. However, the limitation of this study was that the chickens were only grown for two weeks and therefore further research should be conducted by following similar birds to harvest age to see if this therapy does ultimately yield larger healthier birds.

\section{Conclusions}

The recombinant gEGF-expressing Lactococcus lactis could produce the secretory form of gEGF and the expression level of gEGF was associated with the concentration of inducer (nisin) and different induction time. Besides, gEGF stimulated the proliferation of UMNSAH/DF-1 cells, increased growth performance, promoted intestinal development, and improved the immune functioning of broiler chickens. This study may be an indication for the application of gEGF as a growth promotant in commercial broiler breeding. 


\section{Methods}

\section{Bacterial strains, plasmids, medium for bacterial growth}

Lactococcus lactis NZ3900 (NIZO food research B.V., Netherlands) was grown in M17 (Qingdao Hope BioTechnology Co., Ltd.) supplemented with $0.5 \%$ (wt/vol) glucose at $30^{\circ} \mathrm{C}$ without vibration. The plasmid pNZ8149 was obtained from NIZO food research B.V., The Netherlands. Transformed L. lactis cells were selected on M17 without glucose.

\section{Construction of the recombinant plasmid pNZ8149-gEGF and transformation procedures}

The sequence of the mature gEGF peptide was deduced by aligning the amino acid sequence of progallus epidermal growth factor (pro-gEGF; NCBI Reference Sequence: NP_001001292.1) with that of the mature EGF of other species using Clustal Omega (https://www.ebi.ac.uk/Tools/msa/clustalo/) and and the general sequence of EGF-like molecules referred previously [46]. Afterwards, a codon-optimized fusion gene fragment of gEGF and $\mathrm{SP}_{\mathrm{usp} 45}$ carrying the $\mathrm{Nco} \bigotimes / \mathrm{Sac} \rrbracket$ restriction sites and a $6 \times$ His-tag was synthesized by AuGCT Co., Ltd. (Wuhan, China), consisting of 287 base pairs (the sequence can be found in the supplemental information). The synthesized gene was digested with Nco $\mathbb{S} / \mathrm{Sac} \rrbracket$ restriction enzymes (Thermo Fisher Scientific, USA) and inserted into the digested pNZ8149 to construct the recombinant plasmid pNZ8149-gEGF. L. lactis transformation was performed by electroporation at $2.0 \mathrm{kV}$ for 4.0 ms using a Micropulser (Bio-Rad, USA), producing an L. lactis strain that could produce gEGF (LLpNZ8149-gEGF). The recombinant plasmid was verified by PCR with the upstream primer pNZ8149-F: 5囚GATTTCGTTCGAAGGAACTAC-3® and the downstream primer pNZ8149-R: 5》ATCAATCAAAGCAACACGTGC-3囚 as well as by restriction enzyme digestion, and the cloned fragments were verified by sequencing at TIANYI HUIYUAN Co., Ltd. (Wuhan, China), with pNZ8149-F and pNZ8149R.

\section{Expression of recombinant gEGF protein in L. lactis}

The LL-pNZ8149-gEGF strain was inoculated into $5 \mathrm{~mL}$ fresh M17 medium (Qingdao Hope BioTechnology Co., Ltd.; 1:25 dilution). When the $600 \mathrm{~nm}$ optical density of the bacterial cultures reached 0.4, expression of gEGF-His6 fusion protein (gEGF) was induced by adding $10 \mathrm{ng} / \mathrm{mL}$ nisin (Sigma-Aldrich Co., Ltd.). The culture was incubated at $37^{\circ} \mathrm{C}$ without vibration for an additional $6 \mathrm{~h}$. To investigate the 
optimal conditions required for induction, we induced the recombinant strain with different concentrations of nisin $(0,1,2.5,5,10,20$, and $40 \mathrm{ng} / \mathrm{mL})$ for $9 \mathrm{~h}$ and at different times $(0,3,6,9,12,15$, 18,21 , and $24 \mathrm{~h}$ ) with $10 \mathrm{ng} / \mathrm{mL}$ nisin. Afterwards, the cultures were centrifuged at $7500 \times$ at $4{ }^{\circ} \mathrm{C}$ for 15 min and the cell pellets were discarded. To inhibit proteolysis in the supernatant, $1 \mathrm{mM}$ phenylmethylsulfonylfluoride (PMSF; Thermo Fisher Scientific, USA) and $10 \mathrm{mM}$ dithiothreitol (DTT; Thermo Fisher Scientific, USA) were added. Loading buffer (10 $\mu$ l, 5x; Beijing Solarbio Science \& Technology Co., Ltd.) was added to $40 \mu$ l of the supernatant, which was then denatured at $100{ }^{\circ} \mathrm{C}$ for 8 min. Western blot was performed on $20 \mu$ l of the supernatants.

\section{Purification of recombinant $g E G F$ protein from $L$. lactis}

Protein samples were prepared from $300 \mathrm{~mL}$ of the recombinant L. lactis cultures after $6 \mathrm{~h}$ of induction at $30{ }^{\circ} \mathrm{C}$ without vibration. Supernatants obtained by centrifugation at $7500 \times g$ for 20 min at $4{ }^{\circ} \mathrm{C}$ were subjected to precipitation with ammonium sulfate ( $80 \%$, w/v; Sinopharm Chemical Reagent Co. Ltd.). The sample was incubated at $4{ }^{\circ} \mathrm{C}$ with automatic stirring for $2 \mathrm{~h}$ and then centrifuged at $12,000 \times g$ for 30 min at $4{ }^{\circ} \mathrm{C}$. The resultant pellet and floating materials were harvested and dissolved in a binding buffer comprising $500 \mathrm{mM} \mathrm{NaCl}, 20 \mathrm{mM} \mathrm{Na}_{3} \mathrm{PO}_{4}$, and $30 \mathrm{mM}$ imidazole at pH 7.4 and dialyzed overnight using a 1.0-kDa cut-off membrane against the same buffer at $4{ }^{\circ} \mathrm{C}$. The supernatant was centrifuged at 10,000 $\times g$ for 15 min and passed through a $0.45-\mu \mathrm{m}$ filter (Merck Millipore Ltd.) to remove insoluble debris. The supernatant was loaded into a HisTrap HP (GE Healthcare Co., Ltd., UK) column pre-equilibrated with binding buffer and shaken gently at $4{ }^{\circ} \mathrm{C}$ for $60 \mathrm{~min}$. The column was then washed with the native wash buffer (500 mM NaCl, $20 \mathrm{mM} \mathrm{Na}_{3} \mathrm{PO}_{4}$, and $60 \mathrm{mM}$ imidazole at pH 7.4). Finally, the bound protein was eluted with native elution buffer $\left(500 \mathrm{mM} \mathrm{NaCl}, 20 \mathrm{mM} \mathrm{Na}_{3} \mathrm{PO}_{4}\right.$, and $500 \mathrm{mM}$ imidazole at pH 7.4) and analyzed using Tricine-SDS-PAGE. The purified protein was then dialyzed against phosphate-buffered saline (PBS). The concentration of the purified protein was determined using a bicinchoninic acid (BCA) protein assay kit (Thermo Fisher Scientific, USA).

\section{In vitro experiment}

UMNSAH/DF-1 cells, a chicken embryo fibroblast line (BeNa Culture Collection Co., Ltd.), were cultured for $24 \mathrm{~h}$ in Dulbecco's modified Eagle's medium (DMEM; Thermo Fisher Scientific, USA) containing 10\% fetal 
bovine serum (Thermo Fisher Scientific, USA) until they attained a full monolayer, after which they were diluted at 1:10. Next, $100 \mu \mathrm{l}$ of the diluted cell culture was added into each well of a 96 -well Cell Culture Cluster (Corning Inc., China) and cultured for $12 \mathrm{~h}$ and then starved for $12 \mathrm{~h}$ in DMEM without fetal bovine serum ${ }^{\circ} \mathrm{C}$. Afterwards, cell cultures were washed with $100 \mu$ PBS before being treated with $100 \mu$ DMEM containing $1-20 \mu \mathrm{g} / \mathrm{mL}$ of gEGF for $12 \mathrm{~h}$, while the control was treated with $100 \mu \mathrm{l}$ DMEM without gEGF. Finally, $10 \mu \mathrm{l}$ Cell Counting Kit-8 (Dojindo Laboratories) was added to the cell cultures and incubated for 1 h. The $\mathrm{OD}_{450}$ of the cultures was then measured using a MULTISKAN MK3 (Thermo Electron Corporation, USA).

\section{In vivo Animal experiment}

The experimental protocols and the method of euthanasia used were reviewed and approved by the Animal Care Ethics Committee of Huazhong Agricultural University (approval number 42000400002483). The housing, treatment, husbandry, and slaughtering conditions complied with Republic of China guidelines. Sixty-three 5-day-old broilers (Wuhan CP Co., Ltd.) were randomly divided into three groups, each containing 3 replications of 7 broilers (each replication was an experimental unit), and allocated to three different treatments for 14 days: 1) M17 medium (C, as control), 2) supernatant of the LL-pNZ8149 fermentation product (P-LL), and 3) supernatant of the LL-pNZ8149-gEGF fermentation product (gEGF-PLL). The initial average body weight of each group was $91.43 \pm 2.65 \mathrm{~g}, 92.00 \pm 1.98,91.42 \pm 1.51$, respectively (data was presented as mean $\pm S D$ ). The supernatant was prepared by centrifuging the fermentation product at $10,000 \times g$ for $15 \mathrm{~min}$ and discarding the bacterial pellet. Thereafter, all broilers in three groups were orally gavaged with $1 \mathrm{~mL}$ each of their respective treatment agent: either $\mathrm{M}-17$ Control, P-LL, or gEGF-P-LL (2.6 $\mu \mathrm{g}$ gEGF) once a day. All chickens were separately fed in a metal cage with enough corn-soybean meal and were provided ad libitum access to water and feed for the 14-day experimental period. Chickens were reared in an environmentally regulated nursery house in Huazhong Agricultural University. Table 5 provides a list of the exact feed formulation and nutrient composition. 
Feed intake and body weights were recorded every 7 days. All of the broilers were healthy throughout the trial. On day 15, 3 broilers from each cage were randomly selected ( 9 broilers from 3 cages for each treatment) for the collection of samples to evaluate the effect of gEGF on their immune function and intestinal development. Blood was collected from the wing vein of all randomly selected broilers before they were euthanized for the sample collection of intestinal tissues and immune organs. Care and maintenance of all animals were in line with the standards of Institutional Animal Care.

Table 5. Composition of experimental diets.

\begin{tabular}{ll}
\hline Items & Content (\%) \\
\hline Ingredients (\%) & \\
Corn, yellow & 58.10 \\
Soybean meal, dehulled (CP, 48\%) & 30 \\
Fish meal & 4.94 \\
Soybean oil & 3.0 \\
Calcium bicarbonate & 1.4 \\
Limestone & 1.4 \\
Salt & 0.25 \\
L-Lysine-HCl & 0.03 \\
DL-methionine & 0.2 \\
Choline chloride & 0.13 \\
Antioxidant & 0.05 \\
Mineral and vitamin premix & 0.05 \\
Total & 100 \\
& \\
Analyzed nutrition value & \\
Crude protein (\%) & 21 \\
Crude fiber (\%) & 4.95 \\
Calcium (\%) & 0.95 \\
Phosphorus (\%) & 0.62 \\
Lysine (\%) & 1.30 \\
Methionine (\%) & 0.55 \\
Met+Cys (\%) & 0.90 \\
ME (kcal/kg) & 3100 \\
\hline
\end{tabular}

${ }^{\text {a }}$ Supplied per kilogram.

Diet: zinc, 60 mg; iron, 95 mg; copper, 10 mg; iodine, 0.35 mg; selenium, 0.3 mg; manganese, 80 mg; vitamin A, 12,000 IU; vitamin D3,

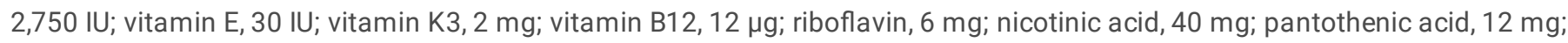
pyridoxine, $3 \mathrm{mg}$; biotin, $0.2 \mathrm{mg}$. 
The collected blood samples were centrifuged at $260 \times g$ at $4{ }^{\circ} \mathrm{C}$ to obtain cell pellets. Thymus, spleen, and bursa of Fabricius were collected from the animals after they were euthanized. The intestinal tract samples of the duodenum, jejunum, and ileum were opened longitudinally, flushed with precooled PBS, and blotted to remove excess fluid. Dissected samples were fixed with $4 \%$ paraformaldehyde (Biosharp Co., Ltd.) for paraffin section preparation to evaluate villus-crypt morphology. A 5-cm section of each tissue from the intestinal tract was scraped with a scalpel to obtain mucosal samples. Mucosal scrapings were placed on pre-weighed cryovials, weighed, and frozen in liquid nitrogen for identification of mucosal slgA. Intestinal samples were embedded in paraffin, sectioned ( $3 \mu \mathrm{m}$ thickness), stained with hematoxylin and eosin for panoramic scanning, and imaged with a Panoramic MIDI slide scanner (3D HISTECH Co., Ltd, Hungary). Villus height and crypt depth were measured from 8 appearance-intact villi with Image-pro plus 6.0 (Media Cybernetics, Inc., Rockville, MD, USA). Frozen mucosa samples were homogenized on ice in saline using a Pro 200 homogenizer (PRO Scientific Co., Ltd., USA). Serum IgA and IgG and small intestinal mucosa (slgA) were measured using ELISA kits (Shanghai Bogoo Biotechnology Co., Ltd.).

\section{Statistical analysis}

The in vivo experimental model used was a completely randomized design with 3 treatments, each containing 3 replications of 7 broilers. Each replication containing 7 broilers was an experimental unit. Data from table 1 represented mean of 3 replications per treatment with 7 broilers per replication and data from table 2, 3 and 4 represented mean of 3 replications per treatment with 3 broilers per replication. All data were analyzed using the statistical program SPSS 19 (IBM Corp., Armonk, NY). The data were analyzed by 1-way ANOVA using Fisher's protected LSD test for multiple comparisons. Results were regarded as significant when $\mathrm{P}<0.05$.

\section{Abbreviations}

gEGF: gallus epidermal growth factor; LL-pNZ8149: Lactococcus lactis transformed with plasmid pNZ8149; LL-pNZ8149-gEGF: Lactococcus lactis that secretes gEGF; P-LL: treatment group fed supernatant of LL-pNZ8149 fermentation product; gEGF-P-LL: treatment group fed supernatant of LLpNZ8149-gEGF fermentation product; EGFR: epidermal growth factor receptor; pEGF: porcine epidermal 
growth factor; mEGF: mouse epidermal growth factor; hEGF: human epidermal growth factor; IBW: initial body weight; BW: body weight; ADG: average daily feed intake.

\section{Declarations}

\section{Ethics approval and consent to participate}

The experimental protocols and the method of euthanasia used were reviewed and approved by the Animal Care Ethics Committee of Huazhong Agricultural University (approval number 42000400002483). The housing, treatment, husbandry, and slaughtering conditions complied with Republic of China guidelines.

\section{Consent for publication}

Not applicable.

\section{Availability of data and material}

The dataset supporting the conclusions of this article is included within the article.

\section{Competing interests}

The authors declare that they have no competing interests.

\section{Funding}

This research was supported by the National Key Research and Development Program of China (2017YFD0501001). The funding source did not participate in the design of the study, collection, analysis, and interpretation of data or in writing the manuscript.

\section{Authors' contributions}

YZ implemented this study and wrote the manuscript; $Y Z, P C$, and SS provided assistance in sample collection; YZ, YX, DS, ZZ, ZL, and XL designed the study. All authors have read and approved the manuscript

\section{Acknowledgements}

The authors would like to thank Biping Li, Yinghui Gong, Yuxuan Hong, and Yucheng He from Huazhong Agricultural University for their assistance with broiler feeding.

\section{Authors' Information}

YZ and SS hold master's degrees and are affiliated with Huazhong Agricultural University. PC holds a bachelor's degree and is affiliated with Huazhong Agricultural University. XL is a doctor from Huazhong 
Agricultural University. DS and ZL are professors from Huazhong Agricultural University. ZZ and YX are associate professors from Huazhong Agricultural University.

\section{References}

1. Cohen S: Isolation of a mouse submaxillary gland protein accelerating incisor eruption and eyelid opening in the new-born animal. J Biol Chem 1962, 237.

2. Klingensmith NJ, Yoseph BP, Liang Z, Lyons JD, Burd EM, Margoles LM, Koval M, Ford ML, Coopersmith CM: Epidermal growth factor improves intestinal integrity and survival in murine sepsis following chronic alcohol ingestion. Shock 2017, 47.

3. Grupcev G, Wallin C, Emås S, Theodorsson E, Hellström PM; Transforming growth factor-alpha and epidermal growth factor inhibit gastric acid secretion and stimulate release of somatostatin and neurotensin in the conscious rat. Regul Pept 1994, 52(2), 111.

4. Schultz GA, Heyner S: Growth factors in preimplantation mammalian embryos. Oxford Rev Reprod B 1993, 15: 43.

5. Dadi TD, Li MW., Lloyd KC: EGF and TGF-alpha supplementation enhances development of cloned mouse embryos. Cloning Stem Cell 2007, 9(3): 315-326.

6. Ledeganck KJ, Boulet GA, Bogers JJ, Verpooten GA, De WBY, Chulso M: The TRPM6/EGF pathway is downregulated in a rat model of cisplatin nephrotoxicity. PLOS ONE 2013, 8(2): e57016-.

7. Pavlov TS, Levchenko V, O'Connor PM, llatovskaya DV, Palygin O, Mori T, Mattson DL, Sorokin A, Lombard JH, Cowley AWJr, Staruschenko A: Deficiency of renal cortical egf increases enac activity and contributes to salt-sensitive hypertension. J Am Soc Nephrol 2013, 24(7): 1053-1062.

8. Bracher A, Cardona AS, Tauber S, Fink AM, Steiner A, Pehamberger H, Niederleithner $\mathrm{H}$, Petzelbauer $\mathrm{P}$, Gröger M, Loewe R: Epidermal growth factor facilitates melanoma lymph node metastasis by influencing tumor lymphangiogenesis. J Invest Dermatol 2013, 133(1): 230-238.

9. Liao YH, Chiang KH, Shieh JM, Huang CR, Shen CJ, Huang WC, Chen BK: Epidermal growth factorinduced ANGPTL4 enhances anoikis resistance and tumour metastasis in head and neck squamous cell carcinoma. Oncogene

10. Oka T, Sakamoto S, Miyoshi K, Fuwa T, Yoda K, Yamasaki M, Tamura G, Miyake T: Synthesis and secretion of human epidermal growth factor by Escherichia coli.. Proc Nat Acad Sci 1985, 82(21): 7212-7216.

11. Kim DG, Min MK, Ahn SC, Kim JK, Kong IS: Expression of a fusion protein containing human epidermal growth factor and the collagen-binding domain of vibrio. Biotechnol Lett 2009, 31(2): 259264.

12. Yamagata H, Nakahama K, Suzuki Y, Kakinuma A, Tsukagoshi N, Udaka S: Use of Bacillus brevis for efficient synthesis and secretion of human epidermal growth factor. Proc Natl Acad Sci 1989, 86(10): 3589-3593. 
13. Ebisu S, Takagi H, Kadowaki K, Yamagata H, Udaka S: The efficient production of human epidermal growth factor by Bacillus brevis. Ann NY Acad Sci 1996, 782(1): 115-122.

14. Urdea MS, Merryweather JP, Mullenbach GT, Coit D, Heberlein U, Valenzuela P, Barr PJ: Chemical synthesis of a gene for human epidermal growth factor urogastrone and its expression in yeast. Proc Natl Acad Sci 1983, 80(24): 7461-7465.

15. 15Brake AJ, Merryweather JP, Coit DG, Heberlein UA, Masiarz FR, Mullenbach GT, Urdea MS, Valenzuela P, Barr PJ: a-Factor-directed synthesis and secretion of mature foreign proteins in Saccharomyces cerevisiae. Proc Nat Acad Sci 1984, 81(15): 4642 -4646】

16. Bedford A, Huynh E, Fu M, Zhu C, Wey D, De Lange C, Li JL: Growth performance of early-weaned pigs is enhanced by feeding epidermal growth factor-expressing Lactococcus lactis fermentation product. J Biotechnol 2014, 173: 47-52.

17. Lee DN, Kuo TY, Chen MC, Tang TY, Liu FH, Weng CF: Expression of porcine epidermal growth factor in Pichia pastoris and its biology activity in early-weaned piglets. Life Sci 2006, 78(6): 0-654.

18. Elliott SN, Wallace JL, Mcknight W, Gall, DG, Hardin JA, Olson M, Buret A: Bacterial colonization and healing of gastric ulcers: the effects of epidermal growth factor. Am J Physiol Gastrointest Liver Physiol 2000, 278(1): G105.

19. Lambrosteski JM, Kalischuk LD, Inglis GD, Buret AG: Epidermal growth factor inhibits Campylobacter jejuni-induced claudin-4 disruption, loss of epithelial barrier function, and Escherichia coli. translocation. Infect Immun 2008, 76(8): 3390-3398.

20. Donovan SM, Zijlstra RT, Odle J: Use of the piglet to study the role of growth factors in neonatal intestinal development. Endocr Regul 1994, 28(4): 153-162.

21. Kim E, Leung H, Akhtar N, Li J, Barta, JR, Wang Y, Yang C, Kiarie E: Growth performance and gastrointestinal responses of broiler chickens fed corn-soybean meal diet without or with exogenous epidermal growth factor upon challenge with Eimeria. Poult Sci 2017, 96(10): 3676.

22. Shimizu N, Fukuzono S, Harada Y, Fujimori K, Gotoh K,Yamazaki Y: Mass production of human epidermal growth factor using fed-batch cultures of recombinant Escherichia coli.. Biotechnol Bioeng 2010, 38(1): 37-42.

23. Mierau I, Olieman K, Mond J, Smid EJ: Optimization of the Lactococcus lactisnisin-controlled gene expression system nice for industrial applications. Microb Cell Fact 2005, 4(1): 16-0.

24. Brink BT, Otto R, Hansen UP, Konings WN: Energy recycling by lactate efflux in growing and nongrowing cells of streptococcus cremoris. J Bacteriol 1985, 162(1): 383-90.

25. Gill RT: Genomic analysis of high-cell-density recombinant Escherichia coli. fermentation and "cell conditioning" for improved recombinant protein yield. Biotechnol Bioeng 2015, 72(1): 85-95.

26. Ozturk AM, Sozbilen MC, Sevgili E, Dagci T, Özyalcin H, Armagan G: Epidermal growth factor regulates apoptosis and oxidative stress in a rat model of spinal cord injury. Injury 2018, 49(6): 1038.

27. Aoyagi T, Kato N, Suya H, Miura Y, Yoshida T, Ohura T: Effects of mouse and human epidermal growth factor on the outgrowing epidermis of pig and human skin explants. J Dermato/ 1984, 11(6): 519-522. 
28. Lax I, Johnson A, Howk R, Sap J, Givol D: Chicken epidermal growth factor (EGF) receptor: cDNA cloning, expression in mouse cells, and differential binding of EGF and transforming growth factor alpha. Mol Cell Biol 1988, 8(5): 1970-1978.

29. Yu A, Matsuda Y, Takeda A, Uchinuma E, Kuroyanagi Y: Effect of EGF and bFGF on fibroblast proliferation and angiogenic cytokine production from cultured dermal substitutes. J Biomater Sci Polym Ed ahead-of-print(ahead-of-print) 2012, 1-10.

30. Clark JA, Lane RH, Maclennan NK, Holubec H, Dvorakova K, Halpern MD, Williams CS, Payne CM, Dvorak B: Epidermal growth factor reduces intestinal apoptosis in an experimental model of necrotizing enterocolitis. Am. J. Physiol. Gastrointest. Liver Physiol 2005, 288(4): G755-G762.

31. Wang $D$, Xu S, Lin Y, Fang Z, Che L, Xue B, Wu D: Recombinant porcine epidermal growth factorsecreting Lactococcus lactis promotes the growth performance of early-weaned piglets. BMC Vet Res 2014, 10(1): 171.

32. Carpenter G, Cohen S: Epidermal growth factor. J Biol Chem 1990, 265: 7709-

33. Bi Q, Zhang Q, Ma J, Xu M, Zhang SJ, Qiu BS, Xia B, Gu HF, Hong JF, Zhao C, Zhu DJ: Effect of combination therapy with alginate dressing and mouse epidermal growth factor on epidermal stem cells in patients with refractory wounds. Chin Med J 2012, 125(2): 257-261.

34. Miettinen PJ, Perheentupa J, Otonkoski T, Lähteenmäki A, Panula P: EGF- and TGF-alpha-like peptides in human fetal gut. Pediatr Res 1989, 26(1): 25-30.

35. Odle J, Zijlstra RT, Donovan SM: Intestinal effects of milkborne growth factors in neonates of agricultural importance. J Anim Sc 1996, 74(10): 2509-22.

36. Kang P, Toms DY, Cheung Q, Gong J, De LK, Li J: Epidermal growth factor-expressing Lactococcus lactis enhances intestinal development of early-weaned pigs. J Nutr 2010, 140(4): 806-811.

37. Shen WH, Xu RJ: Stability of epidermal growth factor in the gastrointestinal lumen of suckling and weaned pigs. Life Sci 1996, 59(3): 0-208.

38. Viveros A, Chamorro S, Pizarro M, Arija I, Brenes A: Effects of dietary polyphenol-rich grape products on intestinal microflora and gut morphology in broiler chicks. Poult Sci 2011, 90(3): 566-578.

39. Thompson JS: Epidermal growth factor inhibits somatostatin-induced apoptosis. J Surg Res 1999, 81: 95-100.

40. Cravens RL, Goss GR, Chi F, De Boer ED, Davis SW, Hendrix SM, Richardson JA, Johnston SL: The effects of necrotic enteritis, aflatoxin b1, and virginiamycin on growth performance, necrotic enteritis lesion scores, and mortality in young broilers. Poul Sci 2013, 92(8): 1997-2004.

41. Prescott JF, Baggot JD: Antimicrobial Therapy in Veterinary Medicine. Antimicrobial Therapy in Veterinary Medicine 1988, 1-10.

42. Hastings PJ, Rosenberg SM, Slack A: Antibiotic-induced lateral transfer of antibiotic resistance. Trends Microbiol 2004, 12(9): 401-404.

43. Salyers AA, Gupta A, Wang Y: Human intestinal bacteria as reservoirs for antibiotic resistance genes. Trends Microbiol 2004, 12(9): 0-416. 
44. Jernberg C, Lofmark S, Edlund C, Jansson JK: Long-term impacts of antibiotic exposure on the human intestinal microbiota. Microbiology 2010, 156(11): 3216-3223.

45. Cotter PD, Stanton C, Ross RP, Hill C. The Impact of Antibiotics on the Gut Microbiota as Revealed by High Throughput DNA Sequencing. Discov Med 2012, 13(70): 193-199.

46. Carpenter $\mathrm{G}$, Cohen S: Human epidermal growth factor and the proliferation of human fibroblasts. $J$ Cell Physio 2010, 88(2): 227-37.

\section{Figures}

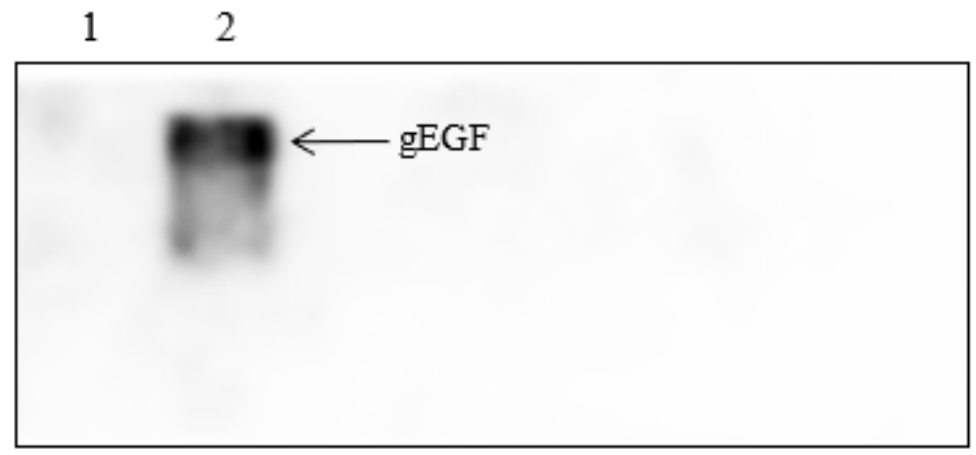

\section{Figure 1}

Analysis of gEGF produced by the recombinant strain. Lanes 1-2: $20 \mu \mathrm{l} /$ well of the supernatant from the LL-pNZ8149 fermentation product and the supernatant from LL-pNZ8149-gEGF fermentation product, respectively. The recombinant gEGF is marked by an arrowhead.

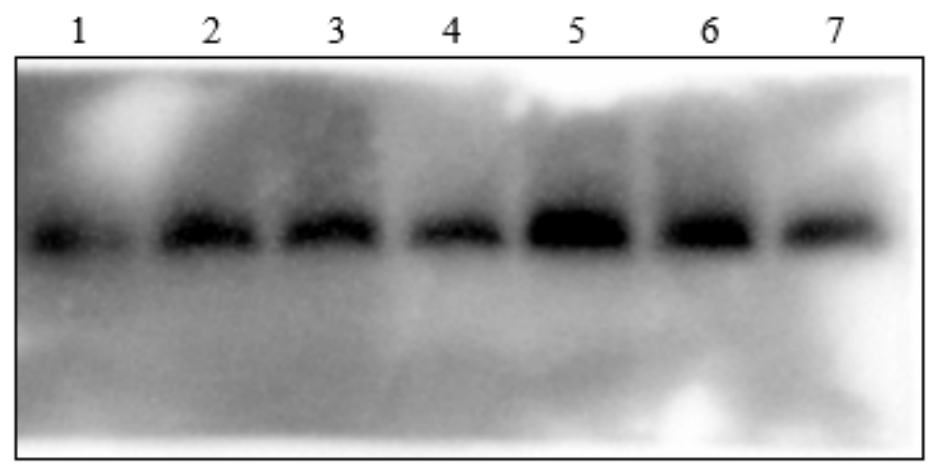

\section{Figure 2}

Analysis of gEGF expression by LL-pNZ8149-gEGF induced with different concentrations of nisin for $9 \mathrm{~h}$. The LL-pNZ8149-gEGF cultures were induced with $0,1,2.5,5,10,20,40 \mathrm{ng} / \mathrm{ml}$ nisin for $6 \mathrm{~h}$ when the optical density of $600 \mathrm{~nm}$ of the bacterial cultures reached 0.4 . Lanes 1-7: $20 \mu \mathrm{l} /$ well of supernatant from each of the induced cultures, respectively. 


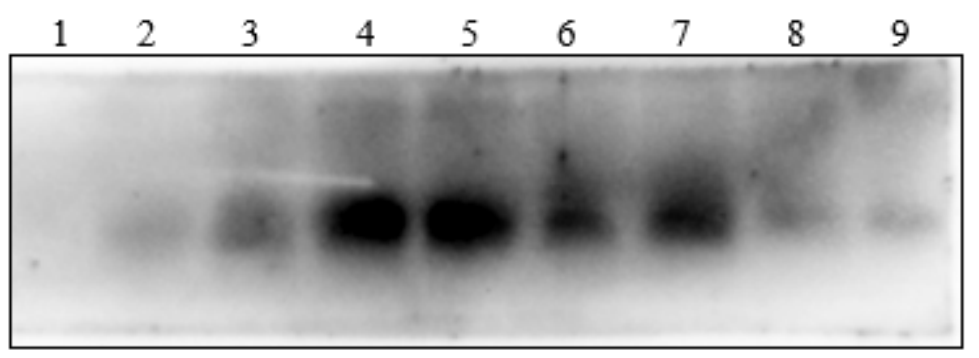

\section{Figure 3}

Analysis of gEGF expression by LL-pNZ8149-gEGF induced for different induction times with $10 \mathrm{ng} / \mathrm{ml}$ nisin. The LL-pNZ8149-gEGF was induced with $10 \mathrm{ng} / \mathrm{ml}$ nisin for $0,3,6,9,12,15,18,21,24 \mathrm{~h}$ when the optical density of $600 \mathrm{~nm}$ of the bacterial cultures reached 0.4. Lanes 1-9: $20 \mu \mathrm{l} /$ well of the supernatant from each of the induced cultures, respectively.

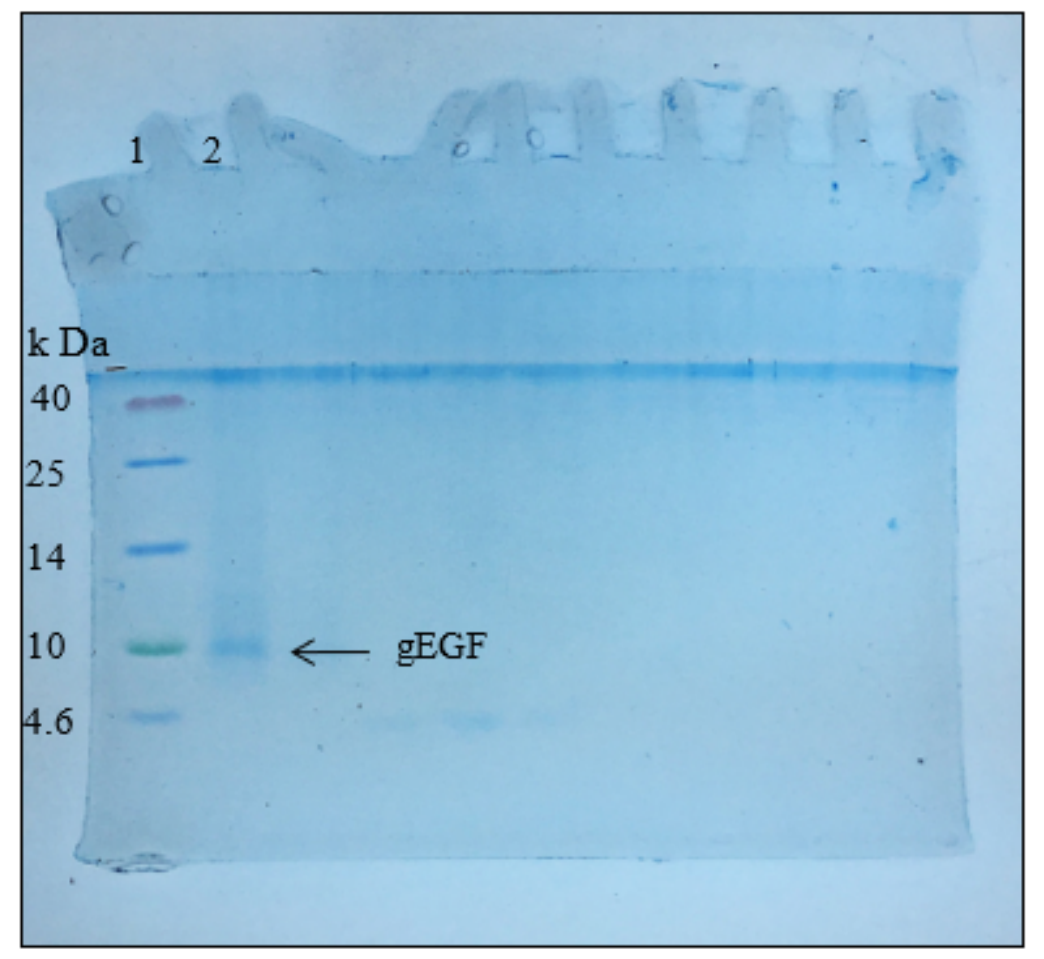

\section{Figure 4}

Analysis of the purified gEGF by Tricine-SDS-PAGE. Lane 1: Spectra Multicolor Low Range Protein Ladder. Lane 2: $20 \mu \mathrm{l} /$ well of protein purified from the supernatant of $300 \mathrm{~mL}$ LL-pNZ8149-gEGF cultures. The recombinant gEGF is marked with an arrowhead at $7 \mathrm{kDa}$. 


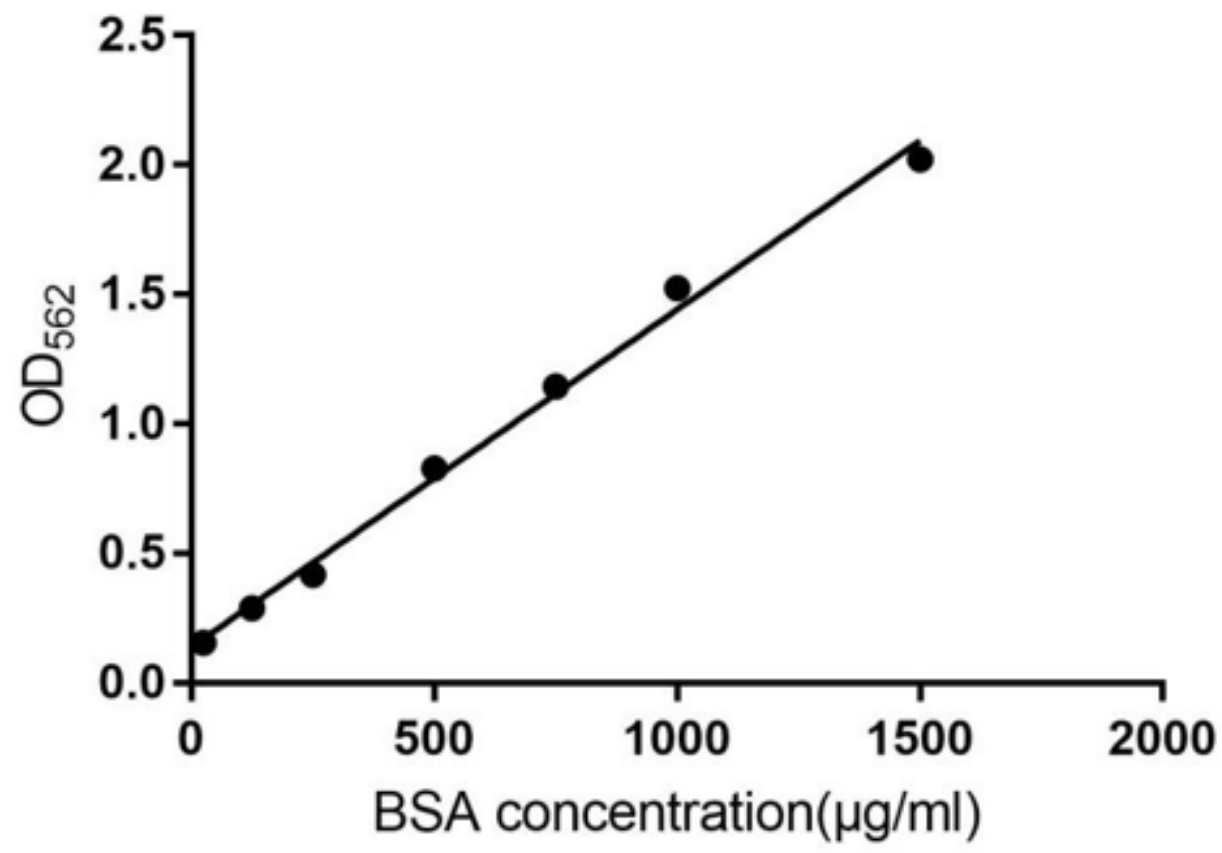

Figure 5

The standard curve for bovine serum albumin (BSA).

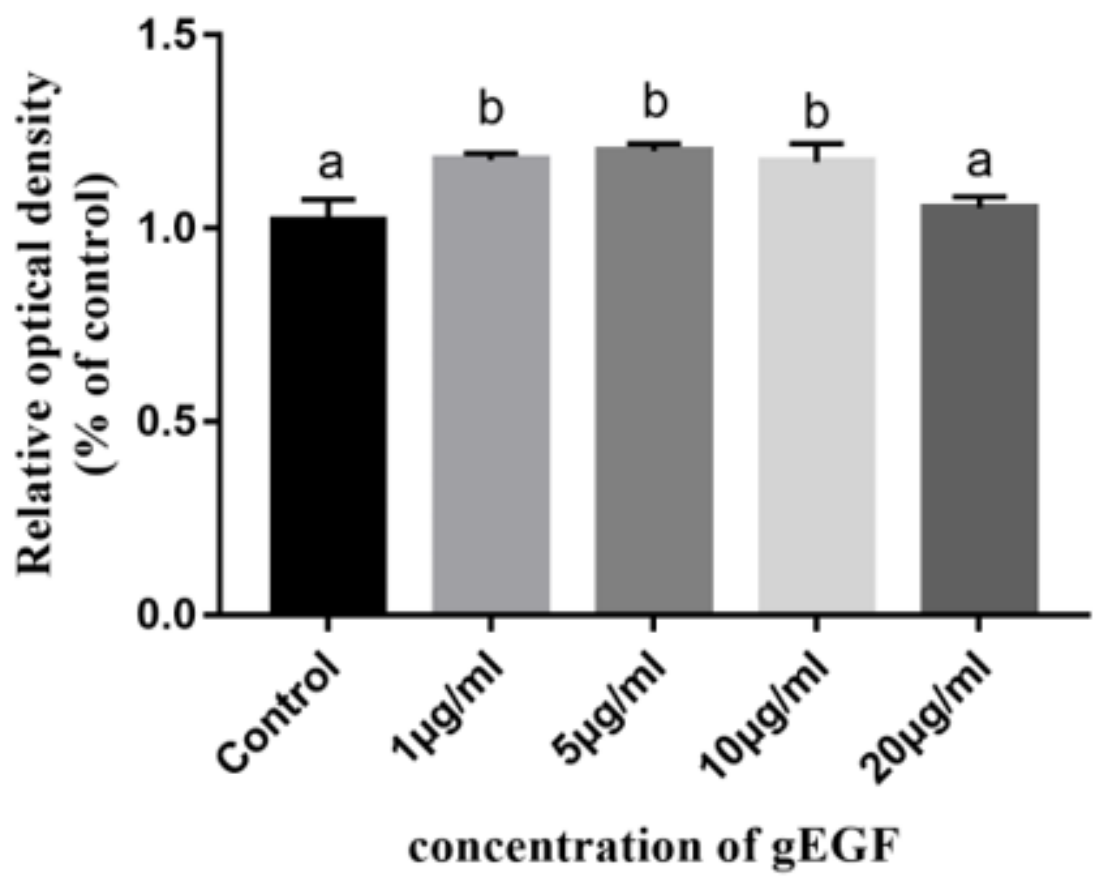

Figure 6

Effect of gEGF of $1-20 \mu \mathrm{g} / \mathrm{mL}$ on DF-1 proliferation. Values are mean $\pm S D(n=5)$. Bars with different letters are significantly different $(p<0.05)$. 


\section{Supplementary Files}

This is a list of supplementary files associated with this preprint. Click to download.

- Supplementarylnformation.doc

- OriginalPictures.docx

- YuZhouNC3RsARRIVEGuidelinesChecklist2014.pdf 